\title{
A Finsler geodesic spray paradigm for wildfire spread modelling
}

\section{Markvorsen, Steen}

\section{Published in:}

Nonlinear Analysis: Real World Applications

Link to article, DOI:

10.1016/j.nonrwa.2015.09.011

Publication date:

2015

\section{Document Version}

Early version, also known as pre-print

Link back to DTU Orbit

Citation (APA):

Markvorsen, S. (2015). A Finsler geodesic spray paradigm for wildfire spread modelling. Nonlinear Analysis: Real World Applications, 28, 208-228. https://doi.org/10.1016/j.nonrwa.2015.09.011

\section{General rights}

Copyright and moral rights for the publications made accessible in the public portal are retained by the authors and/or other copyright owners and it is a condition of accessing publications that users recognise and abide by the legal requirements associated with these rights.

- Users may download and print one copy of any publication from the public portal for the purpose of private study or research.

- You may not further distribute the material or use it for any profit-making activity or commercial gain

- You may freely distribute the URL identifying the publication in the public portal

If you believe that this document breaches copyright please contact us providing details, and we will remove access to the work immediately and investigate your claim 


\title{
A Finsler geodesic spray paradigm for wildfire spread modelling
}

\author{
Steen Markvorsen ${ }^{1}$ \\ DTU Compute, Mathematics, Technical University of Denmark
}

\begin{abstract}
One of the finest and most powerful assets of Finsler geometry is its ability to model, describe, and analyze in precise geometric terms an abundance of physical phenomena that are genuinely asymmetric, see e.g. $[1,2,3,4,5$, $6,7,8,9]$. In this paper we show how wildfires can be naturally included into this family. Specifically we show how the celebrated and much applied Richards' equations for the large scale elliptic wildfire spreads have a rather simple Finsler-geometric formulation. The general Finsler framework can be explicitly 'integrated' to provide detailed - and curvature sensitive - geodesic solutions to the wildfire spread problem. The methods presented here stem directly from first principles of 2-dimensional Finsler geometry, and they can be readily extracted from the seminal monographs [10] and [11], but we will take special care to introduce and exemplify the necessary framework for the implementation of the geometric machinery into this new application - not least in order to facilitate and support the dialog between geometers and the wildfire modelling community. The 'integration' part alluded to above is obtained via the geodesics of the ensuing Finsler metric which represents the local fire templates. The 'paradigm' part of the present proposal is thus concerned with the corresponding shift of attention from the actual fire-lines to consider instead the geodesic spray - the 'fire-particles' - which together, side by side, mold the fire-lines at each instant of time and thence eventually constitute the local and global structure of the wildfire spread.
\end{abstract}

Email address: stema@dtu.dk (Steen Markvorsen)

${ }^{1}$ Telephone: $(+45) 45253049$ 
Keywords: Finsler geometry, wildfire spread, geodesic spray, Richards' equations, Zermelo data, Huyghens' enveloping principle, Hamilton orthogonality, first variation of arc length, indicatrix fields, fire templates, strongly convex ovals.

2000 MSC: Primary 53B40, 58G, 58B20, 58F17.

\section{Introduction}

Every day the World is confronted with wildfires in various regions of our globe. Any wildfire is a highly nonlinear phenomenon, which is in pertinent demand for multidisciplinary and multi-scale analysis and better understanding. Detailed understanding is needed - both for emergency planning, which depends severely on quick and reliable predictions of the wildfire spread in time, as well as for the proper understanding of global issues concerning the $\mathrm{CO}_{2}$ releases and biological and physical changes to the land surface [12]. Such phenomena obviously present scientific opportunities with no shortage of social significance. This fact is repeatedly stressed and documented in every paper that is concerned with the understanding, predicting, and modelling of wildfires, see e.g. [13]. Correspondingly there are several explicit and recent calls from the fire fighter community for new appropriate and effective first principles, i.e. new mathematical models, to handle and understand better the spreading mechanism of the wildfires in forests, grasslands, and wheat fields - with wind, slope, varying fuel properties across the domain and in geographically complicated terrain, see for example the description of the wildfire simulator Prometheus in [14], the comparison of various simulators in $[15,16]$, and the general surveys as in e.g. [17, 18, 19].

\subsection{Outline of paper}

We briefly describe the standard modelling of wildfires including Huyghens' principle in section 2 . In section 3 we emphasise and illustrate how to set up a general fire template field in a parameter domain. The principles of Finsler metrics, the ensuing first variation of arc-length, and the important notion of $F$-geodesics are surveyed in sections 4 and 5 . The resulting $F$-geodesic spray, its enveloping properties, and the induced exponential wildfires are constructed in sections 6 and 7. In sections 8 and 9 the Richards' equations are discussed in terms of their Randers-Zermelo equivalents, and we show that for elliptic wildfires the Richards' equations are solved by the corresponding Finsler-geodesic sprays. Specific examples of $F$-geodesic spray 
driven wildfires are constructed and illustrated in sections 10, 11, and 12 . The final two sections 13 and 14 present the main conclusions from the present paper together with a brief suggestion for further work.

\section{Huyghens' principle}

Following the pioneering works of G. D. Richards [20, 21, 22, 23, 24], van Wagner [25], Anderson et al. [26], and Glasa-Halada [27, 28, 29, 30, 31], we will apply a number of assumptions to be satisfied by the wildfires. We will only consider 2-dimensional, regular, smooth and deterministic wildfires ignited at time $t=0$ on a smooth and regular ignition fireline (or at an ignition point). The fire spread is then represented by a smooth and regular vector function $\gamma(s, t)$ in a $(u, v)$-parameter domain $\mathcal{U} \subset \mathbb{R}^{2}$ so that $\gamma(s, 0)=\eta_{0}(s)$ (the initial fireline) and so that $\gamma(s, t)=\eta_{t}(s)$ is the smooth and regular fireline at time $t>0$. In particular - as part of this assumption we stop the fire before it creates singularities, cut-loci or bear-hugs. In this sense the analysis presented here is only semi-global, but, as we shall see, several global aspects follow naturally already from this outset.

Moreover, we assume that the linearized local spread profile, the so-called firelet, from every point in the fuel domain is known and that it is modelled by a time-invariant and strongly convex oval with the ignition point marked in its interior. This pointed oval field is eventually to be considered as the so-called indicatrix field (see the precise formal definition 4.1 below) for the ensuing Finsler metric via which the wildfires are molded and spread.

Remark 2.1. Time-invariance of the indicatrix field is a strong and not quite realistic condition to assume. Although wildfires usually spread relatively fast the fuel conditions in a given region will clearly change significantly during just 24 hours. We refer to [21] and [32] for the first atttempts to incorporate time-dependent fuel data and meteorological conditions into the enveloping method for elliptic indicatrix fields. (The latter reference seems, however, to build on a mis-interpretation of the first mentioned reference.) In the setting of [21] the elliptic fuel data are only allowed to vary as given functions of time - they are not allowed to vary spatially. We claim that the present Finsler geodesic spray paradigm, that will be unfolded below, can be modified to allow both time and spatial variations not only for the elliptic 
indicatrix fields but for any field of strongly convex pointed ovals. This issue will be taken up in detail in a forthcoming work.

The wildfires under consideration are deterministic in the sense that once such a field of pointed ovals - an indicatrix field - has been chosen in the parameter domain, then there is a unique Huyghens' wildfire in $\mathcal{U}$ which satisfies Huyghens's envelope principle and whose local linearization is precisely the given template field - see Innami's precise statement to that effect below. The Huyghens' principle is the following: Any fire front $\eta_{t_{1}}(s)$ at time $t_{1}$ is the envelope of the point-ignited wildfires of duration $t_{1}-t_{0}$ from the points on the previous fireline $\eta_{t_{0}}(s)$ from time $t_{0}$. In other words, each point on any given initial fire front at time $t_{0}$ can be considered as an ignition point of a small local fire of duration $t_{1}-t_{0}$ which causes the burning out of some area around the ignition point. The resulting fire front at time $t_{1}$ is then given by the envelope of all these burnt out areas. Huyghens' principle is formulated in this setting in e.g. [26] and in its most general version in [33].

In this terminology the fundamental result of N. Innami, which is alluded to above, says essentially:

Theorem 2.2 (N. Innami, [34, 35]). Suppose that every wildfire $\gamma(s, t)=$ $(u(s, t), v(s, t))$ in $\mathcal{U}$ satisfies a second order differential equation system given by two fixed smooth functions of four variables $A^{1}(u, v, x, y)$ and $A^{2}(u, v, x, y)$ in the following sense:

$$
\begin{aligned}
u_{t t}^{\prime \prime}(s, t) & =A^{1}\left(u(s, t), v(s, t), u_{t}^{\prime}(s, t), v_{t}^{\prime}(s, t)\right) \\
v_{t t}^{\prime \prime}(s, t) & =A^{2}\left(u(s, t), v(s, t), u_{t}^{\prime}(s, t), v_{t}^{\prime}(s, t)\right) .
\end{aligned} .
$$

Suppose further that every wildfire spread satisfies Huyghens' principle and is everywhere compatible with the given template field of infinitesimal linearized firelet templates. Then the rays of the spread are the geodesics (extremals) of the Finsler metric determined by the template indicatrix field.

In the following we will explain and illustrate this appearance of a compatible Finsler metric and show how to set up the geodesic spray equations, i.e. how to find the functions $A^{1}$ and $A^{2}$ from the Finsler metric - both in general and in particular examples. 


\section{Parametric domains}

A real world fuel domain in a geographic region is usually not directly given as a flat domain $\mathcal{U}$ in $\mathbb{R}^{2}$. The precise representation of the fuel domain in such a flat parameter domain therefore needs some consideration.

The specific choice of fire template (indicatrix, or pointed oval) at each point $(u, v)$ in the parameter domain depends on the fuel condition, the wind, and the topography (the slope) of the actual real world fuel domain at the corresponding point $r(u, v)$. For example, the slope in figure 1 is given by the simple mapping $r(u, v)=(u, v, \operatorname{erf}(u))$, where $\operatorname{erf}(u)$ is the error function. In practice the choice of fire template field should precisely model and represent the fire line obtained in the real world fuel domain after one time unit started at the origin of the (sloped) tangent plane of the fuel domain at $r(u, v)$ and under the experimental conditions that this tangent plane is equipped with homogeneous conditions, i.e. besides its constant slope, it has constant fuel density and constant wind - the constants represent the respective values at the point $r(u, v)$ in the fuel domain that is being modeled. Equipped in this way each tangent plane then becomes the carrier of the local linearized version of the fuel conditions in the actual fuel domain at the respective points. All this information is then 'pulled back' into the parameter domain $\mathcal{U}$ and represented there via the inverse of the fuel domain map $r$.

For example, the slope in figure 1 is represented in the parameter domain by a so-called Matsumoto field of indicatrices as shown to the right in that figure. The shift of 'center point' towards the left of the ovals shows that the wildfire is locally set to burn faster uphill than downhill. The slopedependence together with the wind- and fuel-properties can be represented by various other choices of oval fields, see figure 2 - the choice in each case must be supported by experiments, observations, and micro-scale physical and chemical analysis of the fuel domain as indicated above. The standard large scale fuel data for a general wildfire is described in much more detail by the Rothermel model $[36,37]$. The prime task of the wildfire modelling community is thence reduced to choosing the best such smoothly varying oval field in the parameter domain in each concrete case - based on local information about the fuel domain in question either from experience and monitoring of previous wildfires or from laboratory experiments as those conducted e.g. by André and Viegas et al. [38, 39, 40]. In this paper we will mainly assume 
that such a choice of template field is constructed - or given - in advance. See also the work of Margerit, Sero-Guillaume et al. [41, 42] concerning the more detailed local and micro-local modelling of fire-phenomena which, on the large scale, reduces to the two-dimensional fire template modelling considered here.

When everything has been set up and represented in the parameter domain the wildfire spread problem can be solved there either numerically or analytically and then eventually it can be lifted back into the real fuel domain by the fuel domain mapping $r: \mathcal{U} \mapsto S \subset \mathbb{R}^{3}$.
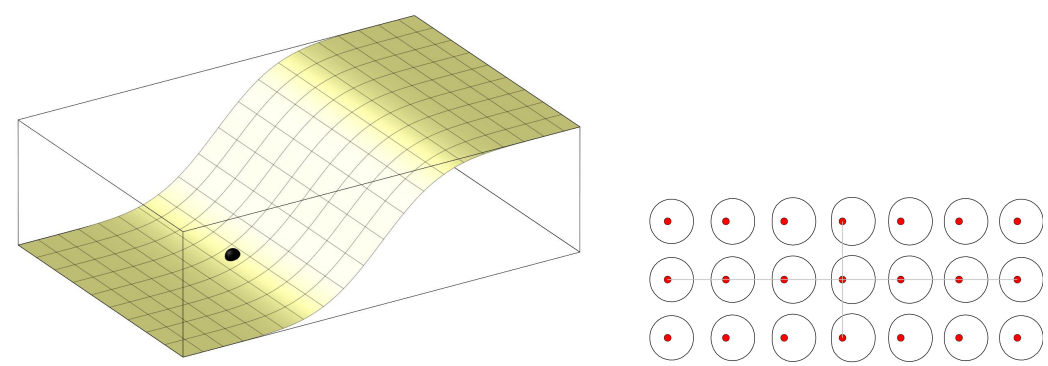

Figure 1: Elementary graph surface $S=r(\mathcal{U})$ and a corresponding slope dependent fire template field in the $(u, v)$-parameter-domain $\mathcal{U}$.
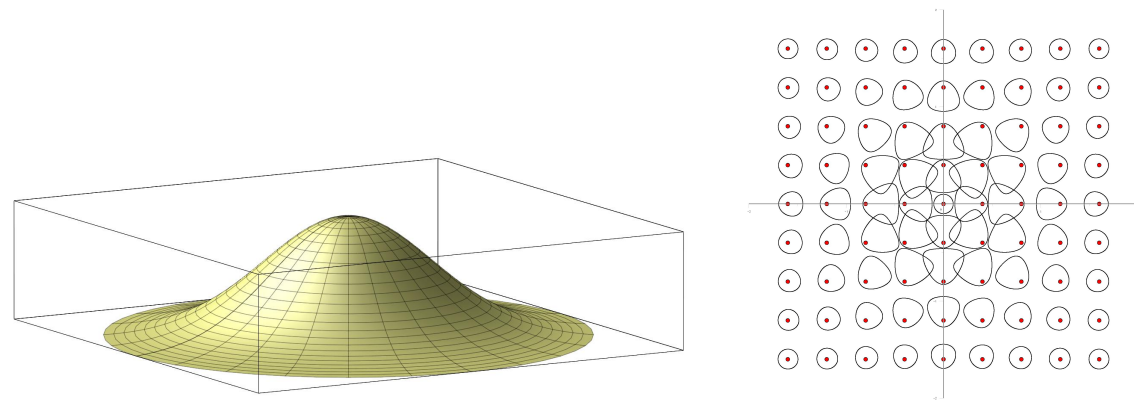

Figure 2: A doubly curved graph surface and a corresponding slope dependent fire template field in the horizontal $(u, v)$-parameter domain. 
We survey the necessary definitions and in particular one standard result (the first variation formula for Finsler arc length) which is very important for setting up the wildfire solutions in the parameter domain.

\section{Finsler metrics}

By classical definition, see [10] and [11], a Finsler metric on a domain $\mathcal{U}$ is a smooth family of Minkowski norms on the tangent planes, i.e. a smooth family of indicatrix templates which in each tangent plane $T_{p} \mathcal{U}$ at the respective points $p=(u, v)$ in the parameter domain $\mathcal{U}$ is determined by a nonnegative function $F$ as follows:

1. $F$ is smooth on the punctured tangent plane $T_{p} \mathcal{U}-\{(0,0)\}$.

2. $F$ is positively homogeneous of degree one: $F(k V)=k F(V)$ for every $V \in T_{p} \mathcal{U}$ and every $k>0$.

3. The following bilinear symmetric form on the tangent space is positive definite:

$$
g_{p, V}(U, W)=\frac{1}{2} \frac{\partial^{2}}{\partial t \partial s}\left[F^{2}(V+s U+t W)\right]_{\mid s=t=0}
$$

Since the function $F$ is homogenous of degree 1, the fundamental metric $g_{p, V}(U, W)$ satisfies the following:

$$
\begin{aligned}
g_{p, V}(V, W) & =\frac{1}{2} \frac{\partial}{\partial t}\left[F^{2}(V+t W)\right]_{\mid t=0} \\
g_{p, V}(V, V) & =F^{2}(V)=\|V\|_{F}^{2} .
\end{aligned}
$$

Suppose that we use the canonical basis $\left\{\partial_{u}=b_{1}, \partial_{v}=b_{2}\right\}$ in $T_{p} \mathcal{U}$, and let $V=x^{i} b_{i}$. Then we can define coordinates of $g=g_{p, V}$ in the usual way:

$$
\begin{aligned}
2 g_{i j}(V) & =2 g_{p, V}\left(b_{i}, b_{j}\right) \\
& =\frac{\partial^{2}}{\partial t \partial s}\left[F^{2}\left(V+s b_{i}+t b_{j}\right)\right]_{\mid s=t=0} \\
& =\operatorname{Hess}_{i j}\left(F^{2}\right)(V) \\
& =\left[F^{2}\right]_{x^{i} x^{j}}(V)
\end{aligned}
$$

where the Hessian is evaluated at the vector $V$ and where the last line $\left[F^{2}\right]_{x^{i} x^{j}}$ is 'shorthand' for the double derivatives of $F^{2}$ with respect to the tangent 
plane coordinates $x^{i}$. We have used the indexed $u^{k}$ to denote the respective coordinates in the domain - in our two-dimensional case: $u^{1}=u$ and $u^{2}=v$ and similarly we will use the indexed $x^{k}$ to denote the coordinates in the tangent planes - in our case: $x^{1}=x$ and $x^{2}=y$.

Since $F$ also depends on the point $p=(u, v)=\left(u^{1}, u^{2}\right)$ we will write the complete information about $F$ presented as a function of 4 variables in either one of the following presentations all with respect to the chosen canonical coordinate system and bases in $\mathcal{U}$ in the parameter domain:

$$
F=F(V)=F(p, V)=F(u, v, x, y)=F\left(u^{1}, u^{2}, x^{1}, x^{2}\right) \quad .
$$

In the following we shall need other partial derivatives of $F^{2}-$ such as $\left[F^{2}\right]_{u^{k}}(V)$ and $\left[F^{2}\right]_{u^{l} x^{k}}(V)$ - as well as the inverse matrix of $g_{i j}(V)$, which are now all well-defined, e.g.:

$$
\left[g^{i j}(V)\right]=\left[g_{i j}(V)\right]^{-1} \quad, \quad \text { so that } \quad\left[g^{i j}(V) g_{k j}(V)\right]=\left[\begin{array}{cc}
1 & 0 \\
0 & 1
\end{array}\right]
$$

The (fire-)templates, the pointed ovals, which have already been alluded to in the introduction are built into a given Finsler metric as the $F$-unit 'circles' in the respective tangent planes - formally they are called indicatrices of the Finsler metric, and they are defined in terms of $F$ as follows:

Definition 4.1. The set of points in the tangent plane $T_{p} \mathcal{U}$ which have $F$ unit position vectors is called the indicatrix of $F$ at $p$ :

$$
\mathcal{I}_{p}=F^{-1}(1)=\left\{V \in T_{p} \mathcal{U} \mid F(V)=1\right\}
$$

Since $g_{p, V}$ is positive definite, the indicatrix $\mathcal{I}_{p}$ is automatically strongly convex in its tangent plane at $p$, and it contains the origin of the tangent plane in its interior, see [11]. It is therefore a pointed oval - the point being that origin of the tangent plane - as needed.

One immediate task is to construct the Finsler metric function $F$ from a given template field consisting of ellipses or other ovals stemming from the pointwise linearized modelling of the wildfire. For example, the usual quadratic equation for a given ellipse cannot be used directly but must be recast into a unique 1-homogeneous version, which then gives the Finsler 
metric $F$ with the given ellipse as its indicatrix. This conversion from template field to Finsler metric is exemplified below, and can be obtained in a number of ways for each given template, see e.g. the so-called Okubo technique in [11, p. 13].

In this sense, then, the fundamental asymmetry of the wildfire phenomenon is already built into the Finsler geometry from the very definition of the metric via the typically asymmetric pointed oval indicatrix templates. This Finsler generalization of the (ordinary, symmetric, and quadratic) Riemannian geometry was in fact envisioned already by Riemann himself [43].

\section{First variation of $\boldsymbol{F}$-arclength}

Following [10, Chapter 5] we survey the derivation of the important first variation formula for the $F$-length functional in a domain $\mathcal{U}$ with a given Finsler metric $F$. It is stated here in its most general ( $n$-dimensional) form

- for notational convenience only - but will be restricted and applied to the two-dimensional cases of wildfires below.

The first variation formula will give us the ODE differential equation conditions for a curve to be an $F$-geodesic in $\mathcal{U}$, i.e. the analytic condition for a curve to be the trace of a fire particle in the 'wildfire' terminology suggested above.

We let $c=c(t)=(u(t), v(t))$ denote a candidate for a geodesic, i.e. a candidate for a fire particle in the parameter domain:

$$
c \quad: \quad[a, b] \rightarrow \mathcal{U} \quad,
$$

and assume that it is a unit speed piecewise $C^{\infty}$ curve in $\mathcal{U}$. This means that

$$
F\left(c(t), c^{\prime}(t)\right)=1 \text { for all } t \in[a, b], \quad \text { and }
$$

there is a partition of $[a, b]$

$$
a=t_{0}<\cdots<t_{m}=b,
$$

so that $c$ is smooth on each subinterval $\left[t_{i-1}, t_{i}\right]$ for every $i=1, \cdots, m$. 
A variation of the curve $c$ is then a piecewise smooth map $H(w, t)$ :

$$
H \quad: \quad(-\varepsilon, \varepsilon) \times[a, b] \rightarrow M
$$

such that

$$
\begin{aligned}
& H \quad \text { is continuous on } \quad(-\varepsilon, \varepsilon) \times[a, b] \\
& H \quad \text { is smooth on each } \quad(-\varepsilon, \varepsilon) \times\left[t_{i-1}, t_{i}\right] \\
& H(0, t)=c(t) \text { for all } \quad a \leq t \leq b
\end{aligned}
$$

The last equation (19) states that $c$ is the base curve in the family of curves $c_{w}(t)=H(w, t)$, which sweep out the variation. The endpoints of $c$ are not necessarily fixed.

The variation $H$ induces the associated so-called variation vector field $W(t)$, so that we have, in local coordinates, using the usual short hand summation convention:

$$
\frac{\partial H}{\partial w}(0, t)=W(t)=\left.W^{k}(t) \frac{\partial}{\partial u^{k}}\right|_{c(t)} \quad .
$$

The $F$-lengths of the individual piecewise smooth curves $c_{w}(t)$ in the variation family $H$ are then given by

$$
\begin{aligned}
\mathcal{L}(w) & =\int_{a}^{b} F\left(c_{w}(t), c_{w}^{\prime}(t)\right) d t \\
& =\sum_{i=1}^{m} \int_{t_{i-1}}^{t_{i}} F\left(c_{w}(t), \frac{\partial H}{\partial t}(w, t)\right) d t .
\end{aligned}
$$

Then we have the following $w$-derivative of $\mathcal{L}(w)$ at $w=0$. Note that $F$ is constant 1 along the base curve. We apply short hand notations such as $\dot{c}=c^{\prime}(t)$ and $\ddot{c}=c^{\prime \prime}(t)$ but suppress the evaluation point $\dot{c}$ from the notation for the partial derivatives e.g. $\left[F^{2}\right]_{u^{l} x^{k}}(\dot{c})$ as in [10, Section 5.1]:

$$
\begin{aligned}
\mathcal{L}^{\prime}(0) & =\int_{a}^{b}\left(\frac{1}{2 F}\right)\left(\left[F^{2}\right]_{u^{k}} W^{k}+\left[F^{2}\right]_{x^{k}} \frac{d W^{k}}{d t}\right) d t \\
& =\frac{1}{2} \int_{a}^{b}\left(\left[F^{2}\right]_{u^{k}}-\left(\frac{d}{d t}\left[F^{2}\right]_{x^{k}}\right)\right) W^{k} d t
\end{aligned}
$$




$$
\begin{gathered}
\quad+\frac{1}{2} \sum_{i=1}^{m}\left[\left[F^{2}\right]_{x^{k}} W^{k}\right]_{t_{i-1}}^{t_{i}} \\
=\frac{1}{2} \int_{a}^{b}\left(\left[F^{2}\right]_{u^{k}}-\left[F^{2}\right]_{u^{l} x^{k}} \dot{c}^{l}-\left[F^{2}\right]_{x^{l} x^{k}} \ddot{c}^{l}\right) W^{k} d t \\
+\sum_{i=1}^{m}\left[g_{j k} \dot{c}^{j} W^{k}\right]_{t_{i-1}}^{t_{i}} \\
=-\int_{a}^{b} g_{j k}\left(\ddot{c}^{j}+2 G^{j}(\dot{c})\right) W^{k} d t+\sum_{i=1}^{m}\left[g_{j k} \dot{c}^{j} V^{k}\right]_{t_{i-1}}^{t_{i}}
\end{gathered}
$$

where the fundamental metric $g_{j k}(x, y)$ has been defined above, and where the geodesic spray coefficients are:

$$
G^{j}(V)=\left(\frac{1}{4}\right) g^{j l}(V)\left(\left[F^{2}\right]_{u^{k} x^{l}}(V) x^{k}-\left[F^{2}\right]_{u^{l}}(V)\right)
$$

There are several consequences of this calculation - including the following which also defines the notion of $F$-geodesics:

Proposition 5.1. If the curve $c$ has fixed end points and minimal length, so that $\mathcal{L}^{\prime}(0)=0$, then $c$ is an F-geodesic, i.e. a smooth curve satisfying the following (geodesic) equations:

$$
\ddot{c}^{j}+2 G^{j}(c, \dot{c})=0 \quad \text { for } \quad j=1,2,
$$

which in our original $2 D$ parameter notation reads as follows:

$$
\begin{aligned}
& u^{\prime \prime}(t)+2 G^{1}\left(u(t), v(t), u^{\prime}(t), v^{\prime}(t)\right)=0 \\
& v^{\prime \prime}(t)+2 G^{2}\left(u(t), v(t), u^{\prime}(t), v^{\prime}(t)\right)=0 .
\end{aligned}
$$

These equations - with $G^{1}$ and $G^{2}$ inserted from (29) - are precisely the $F$-induced equations corresponding to the ODE system in Innami's theorem 2.2 - i.e. $A^{1}=-2 G^{1}$ and $A^{2}=-2 G^{2}$. These $F$-geodesic equations can be numerically solved and displayed as in figures 4 and 11 or, in rare cases, be analytically solved and displayed as in the figures 8 and 9 . 


\section{Enveloping}

The converse to proposition 5.1 also holds - at least locally - in the following sense:

Proposition 6.1. Suppose $c$ is an F-geodesic from a point $p$ to a not too far away point $q$ in $\mathcal{U}$ - i.e. c satisfies the geodesic equation (30) all the way - then $c$ is the F-shortest curve from $p$ to $q$.

The proof of proposition 6.1 does not follow directly from the first variation formula, but involves an application of the so-called exponential map (that we will define and apply also below) together with the Finsler version of the Gauss lemma, see e.g. [11, Chapter 6] or [10, Chapter 11].

The $F$-distance $d_{F}(p, q)$ between two points $p$ and $q$ in a given fuel domain with Finsler metric $F$ can now be defined as follows. This distance is important, because eventually it is to be interpreted as the time it takes the corresponding wildfire to reach the point $q$ when ignited at the point $p$ in the fuel domain.

Definition 6.2. The F-distance from $p$ to $q$ is the length of the shortest geodesic $c_{p, q}$ from $p$ which connects $p$ and $q$ :

$$
d_{F}(p, q)=\mathcal{L}\left(c_{p, q}\right)
$$

If we consider a variation based on an $F$-geodesic and if the endpoints of the geodesic are allowed to move with the variation, then the variation will typically produce curves that have other lengths than the base geodesic, but the derivative of the length function is controlled by the first variation formula for $F$-arc-length which follows directly from the general calculation of $\mathcal{L}^{\prime}(0)$ :

Proposition 6.3. Let $c(s), s \in[a, b]$, denote a (smooth) F-geodesic with $F$-unit speed. Suppose $H$ is a variation of $c$ as above with $H(w, a)=\eta_{a}(u)$ and $H(w, b)=\eta_{b}(u)$. Then

$$
\mathcal{L}^{\prime}(0)=g_{\dot{c}(b)}\left(\dot{c}(b), \dot{\eta}_{b}(0)\right)-g_{\dot{c}(a)}\left(\dot{c}(a), \dot{\eta}_{a}(0)\right)
$$


We interpret the $F$-geodesics from a given point $p$ as fire-particle tracks in the domain - fire tracks that are issuing from the ignition point $p$.

Standard theory for ordinary differential equations gives the existence and uniqueness of these tracks: For any point $p$ in $\mathcal{U}$ and for any $F$-unit vector $V$ in the tangent plane $T_{p} \mathcal{U}$ there exists a unique unit speed $F$-geodesic $c(t), t \in$ $\left[0, T\right.$ issuing from $p=c(0)$ in the direction $V$ so that $c^{\prime}(0)=\left(u^{\prime}(t), v^{\prime}(t)=V\right.$ and $F\left(c(t), c^{\prime}(t)\right)=F\left(u(t), v(t), u^{\prime}(t), v^{\prime}(t)\right)=1$ for all $t \in[0, T[$.

Definition 6.4. The map from $T_{p} \mathcal{U}$ into $\mathcal{U}$ defined from these ingredients is called the exponential map:

$$
\begin{gathered}
\exp _{p}: T_{p} \mathcal{U} \rightarrow \mathcal{U} \\
\exp _{p}(t V)=c_{p, V}(t) \quad, \quad\|V\|_{F}=1,
\end{gathered}
$$

where $c_{p, V}(t)$ is the unique $F$-geodesic starting at $p$ and having initial direction $V$. In particular $\exp _{p}(0)=p$.

The system of fire tracks from $p$ may thus be thought of as images of straight half lines from the tangent plane into the domain via the exponential map.

A $p$-centered $F$-geodesic disk of radius $\rho$ in $\mathcal{U}$ is then defined as the exponential image of the corresponding disk $B_{p}(\rho)$ of $F$-radius $\rho$ in $T_{p} \mathcal{U}$. By definition $B_{p}(\rho)$ is just the $\rho$-scaled version of the indicatrix $\mathcal{I}_{p}$ in the tangent plane at the point $p$. The $\rho$-disk $D_{p}(\rho)$ in $\mathcal{U}$ can be thought of as the domain that has been burnt out by the total system of fire particles issuing from $p$ during the time from $t=0$ to $t=\rho$ :

Definition 6.5. The F-geodesic (fire) disk of radius $\rho$ in $\mathcal{U}$ is defined by

$$
D_{p}(\rho)=\exp _{p}\left(B_{p}(\rho)\right)
$$

and the corresponding fire line $L_{p}(\rho)$ at time $\rho$ is thence the boundary of the fire disk:

$$
L_{p}(\rho)=\partial \exp _{p}\left(B_{p}(\rho)\right)=\exp _{p}\left(\partial B_{p}(\rho)\right) \quad .
$$


The examples in sections 10, 11, and 12 below will show a number of fire discs $D_{p}(\rho)$ for fixed $p$ so that the corresponding fire lines and their spread governed by the exponential map is clearly visualized.

In order to set up the proper Huyghens' principle in the fire particle scenario we finally also need to define the (forward) geodesic $\rho$-envelope of a given curve $\eta_{0}(s)$ in $\mathcal{U}$ :

Definition 6.6. Let $\eta_{0}(s)$ denote a smooth closed curve in $\mathcal{U}$ with a well defined interior (the burnt domain). The F-geodesic (fire) disks of radius $\rho$ based at points on $\eta_{0}$ then cover an annular region around $\eta_{0}$ :

$$
\mathcal{A}_{\rho, \eta_{0}}=\bigcup_{p \in \eta_{0}} D_{p}(\rho)
$$

which may thence be characterized as the points in $\mathcal{U}$ which have $F$-distance $\rho$ or less to $\eta_{0}$ :

$$
\mathcal{A}_{\rho, \eta_{0}}=\left\{q \in \mathcal{U} \mid d_{F}(p, q) \leq \rho \text { for some } p \in \eta_{0}\right\}
$$

The envelope of the fire discs $D_{\eta_{0}}(\rho)$ is now the boundary:

$$
\begin{aligned}
\mathcal{E}_{\rho, \eta_{0}} & =\partial \mathcal{A}_{\rho, \eta_{0}} \\
& =\partial\left\{q \in \mathcal{U} \mid d_{F}(p, q) \leq \rho \text { for some } p \in \eta_{0}\right\} .
\end{aligned}
$$

The envelope therefore consists of those points $q$ in $\mathcal{U}$ which have Finsler distance $\rho$ to some point $p$ on $\eta_{0}$ as well as Finsler distance not less than $\rho$ to all other points on $\eta_{0}$. This envelope has two components in the twodimensional domain $\mathcal{U}$ - one in the interior (burnt out) region bounded by $\eta_{0}$ and one in the non-burnt (out-side) fuel domain defined by $\eta_{0}$. The latter component will be called the forward envelope of the geodesic fire disks based on $\eta_{0}$.

\section{Finsler induced wildfires}

With these ingredients we are now ready to define formally how a given template field induces a unique wildfire spread from a given ignition line or ignition point which is in accordance with Huyghens' enveloping principle. 
Definition 7.1. Let $\mathcal{I}_{p}, p \in \mathcal{U}$, denote a given fire template field in $\mathcal{U}$ with induced Finsler metric $F$ and let $\eta_{0}$ denote a simple closed regular curve in $\mathcal{U}$. Then the $\mathcal{I}$-induced wildfire spread from the ignition curve $\eta_{0}$ is the net $\gamma(s, t)$ defined by the F-geodesic exponential map:

$$
\gamma(s, t)=\exp _{\eta_{0}(s)}(t \cdot V(s)),
$$

where $V(s)$ is the unique $F$-unit vector in the tangent plane $T_{\eta_{0}(s)} \mathcal{U}$ which is $F$-orthogonal to $\eta_{0}^{\prime}(s)$ and points to the right hand side of $\eta_{0}^{\prime}(s)$.

Remark 7.2. This defines the wildfire to only the right hand out-side of $\eta_{0}$. Changing the orientation of $\eta_{0}$ makes the wildfire spread into the left hand in-side of $\eta_{0}$. In the case where $\eta_{0}$ is a very small closed curve, we may as well replace it with a point and the corresponding point-ignited wildfire spread follows accordingly. The above definition of a wildfire spread is the most general definition which of course includes the wildfire spreads induced form the elliptic indicatrix fields. Examples of a fire template fields for various settings are on display in figures 1 and 2.

We observe, that Huyghens' principle is indeed satisfied by this definition and thence it follows from Innami's theorem that there are no other 2.nd order wildfires than the ones determined by the defining equation (42):

Proposition 7.3. Huyghens' principle is satisfied by the wildfires that are generated by the F-geodesic spray and represented by the exponential map in (42).

Proof. Let $\eta_{0}(s)=\gamma\left(s, t_{0}\right)$ and let $\eta_{1}(s)$ denote the envelope of radius $\delta=$ $t_{1}-t_{0}$ in the forward direction from $\eta_{0}$. We must show that $\eta_{1}(s)=\gamma\left(s, t_{1}\right)$. Let $q \in \eta_{1}(s)$. Then $q=\exp _{p}(\delta v)$ for some $p \in \eta_{0}(s)$ and for some $F$ unit forward pointing vector $v$. Suppose that $v$ is not $F$-orthogonal to $\eta_{0}(s)$. Then according to proposition 6.3 there is a variation $W$ which will produce a shorter connection from $\eta_{0}(s)$ to $q$, that is, shorter than $\delta$, and thence also a shorter geodesic from $\eta_{0}(s)$ to $q$. This is a contradiction to the assumption that $q$ is in the $\delta$ envelope from $\eta_{0}(s)$. Therefore $v$ is $F$-orthogonal to $\eta_{0}(s)$. Moreover, the geodesic $\exp _{p}\left(\left(t-t_{0}\right) v\right)$ is also $F$-orthogonal to $\eta_{1}(s)$ because otherwise there would now be a shorter than $\delta$ geodesic from $p$ to $\eta_{1}(s)$ which is again a contradiction. In total it follows that $\exp _{p}\left(\left(t-t_{0}\right) v\right)$ is precisely the geodesic $\gamma\left(s_{0}, t\right), t \in\left[t_{0}, t_{1}\right]$, for some $s_{0}$, and that $\eta_{1}(s)=\gamma\left(s, t_{1}\right)$, so 
that the Huyghens' envelope construction in definition 6.6 is identical to the forward spread of $\eta_{0}(s)$ obtained by extending the $F$-orthogonal geodesics by the amount of $\delta$ in the forward direction from $\eta_{0}$ in accordance with (42).

It follows directly from the above proof that every wildfire $\gamma(s, t)$ is therefore forming the Finsler version of what is classically called a geodesic parallel net, see [44, Section 4-3 p. 136] and [45, Lemma 4.3.6 p. 80 and Def. 4.3.7 p. 81].

Moreover, we can extract from the same proof the following Hamilton orthogonality at every point in the net - see [46, Section 3].

Corollary 7.4. Every wildfire defined by (42) satisfies everywhere the socalled Hamilton orthogonality conditions with respect to the Finsler metric F:

$$
\begin{array}{rll}
\left\|\gamma_{t}^{\prime}(s, t)\right\|_{F} & = & 1 \\
\gamma_{s}^{\prime}(s, t) & \perp_{F} & \gamma_{t}^{\prime}(s, t),
\end{array}
$$

where $V \perp_{F} W$ is defined in the obvious way:

$$
g_{p, V}(V, W)=\frac{1}{2} \frac{\partial}{\partial t}\left[F^{2}(V+t W)\right]_{\mid t=0}=0
$$

Conversely, via existence and uniqueness of solutions to the PDE system (43), (44), these equations are equivalent to the geodesic ODE equations (31) and (32).

Remark 7.5. The equation (43) is the precise version of what we have previously referred to as compatibility of the wildfire with the Finsler metric and thus with the given indicatrix field.

The local Hamilton orthogonality is illustrated in figure 3. The equation $g_{p, V}(V, W)=0$ simply means that the vector $W$ is parallel to the tangent of the indicatrix $\mathcal{I}_{p}$ at the point on $\mathcal{I}_{p}$ which has $V$ as its position vector. 


\section{The Randers-Zermelo elliptic wildfires}

We consider a surface $S$ in $\mathbb{R}^{3}$ with parametrization $r(u, v)$ so that $r$ : $\mathcal{U} \mapsto S$, as for example in figures 1,2 , and 6 .

As part of our general assumptions, all fuel information on the surface is intrinsically encoded into a pointed oval, an indicatrix, in each tangent plane $T_{(u, v)} \mathcal{U}$ at the point $(u, v)$ of the parameter domain. In this section we assume that all indicatrices are pointed ellipses. In practice they are found and determined in the way already described in the introduction. Finsler metrics with elliptic indicatrix fields are called Randers metrics after G. Randers, [47].

For the elliptic indicatrix fields we use the notation and description, which is originally and usually applied by the wildfire modelling community, see e.g. [20], [28]. In this setting, the representing ellipse field $\mathcal{I}_{p}=E_{(u, v)}$ is parametrized as follows in the tangent space basis $\left\{\partial_{u}, \partial_{v}\right\}$ at $(u, v)$ in the parameter domain:

$$
E_{(u, v)}(\psi)=\left(R_{\theta(u, v)}\left[\begin{array}{c}
a(u, v) \cos (\psi) \\
b(u, v) \sin (\psi)
\end{array}\right]\right)+\left[\begin{array}{c}
c_{1}(u, v) \\
c_{2}(u, v)
\end{array}\right]
$$

where $R_{\theta(u, v)}$ denotes the rotation in the tangent plane at $(u, v)$ by the angle $\theta(u, v)$ in the clock-wise direction, see figure 4 :

$$
R_{\theta(u, v)}=\left[\begin{array}{cc}
\cos (\theta(u, v)) & \sin (\theta(u, v)) \\
-\sin (\theta(u, v)) & \cos (\theta(u, v))
\end{array}\right]
$$

The translation vector $C(u, v)=\left(c_{1}(u, v), c_{2}(u, v)\right)$ (which is contributing to modelling the influence of the wind on each fire template) is always assumed to be sufficiently small so that the resulting rotated and translated ellipse contains the origin of the tangent plane of the parameter domain at the point $(u, v)$, that is, so that the ellipse with its origin becomes a pointed oval in the sense of general Finsler indicatrices.

For the ellipse field models we will apply the so-called Zermelo representation, which is convenient for the analysis to be carried out below. Before translation by the wind vector $C(u, v)$, the ellipse

$$
\left[\begin{array}{l}
x(\psi) \\
y(\psi)
\end{array}\right]=R_{\theta(u, v)}\left[\begin{array}{c}
a(u, v) \cos (\psi) \\
b(u, v) \sin (\psi)
\end{array}\right]
$$


satisfies the classical quadratic equation $Q(x, y)=1$, where

$$
\begin{aligned}
Q(x, y) & =\left(\frac{x \cos (\theta(u, v))-y \sin (\theta(u, v))}{a(u, v)}\right)^{2} \\
& +\left(\frac{x \sin (\theta(u, v))+y \cos (\theta(u, v))}{b(u, v)}\right)^{2}
\end{aligned}
$$

that is:

$$
\left[\begin{array}{ll}
x & y
\end{array}\right] h_{(u, v)}\left[\begin{array}{l}
x \\
y
\end{array}\right]=1
$$

where $h_{(u, v)}$ denotes (half of) the Hessian of the quadratic form:

$$
\begin{aligned}
h_{(u, v)} & =\frac{1}{2} \operatorname{Hessian}(Q(x, y)) \\
& =\frac{1}{a^{2} b^{2}}\left[\begin{array}{cc}
a^{2} \sin ^{2}(\theta)+b^{2} \cos ^{2}(\theta) & \left(a^{2}-b^{2}\right) \sin (\theta) \cos (\theta) \\
\left(a^{2}-b^{2}\right) \sin (\theta) \cos (\theta) & a^{2} \cos ^{2}(\theta)+b^{2} \sin ^{2}(\theta)
\end{array}\right]
\end{aligned}
$$

In passing we observe for later use, that if $\theta=0$ we get the following:

$$
h_{(u, v)}=\frac{1}{a^{2} b^{2}}\left[\begin{array}{cc}
b^{2} & 0 \\
0 & a^{2}
\end{array}\right]=\left[\begin{array}{cc}
\frac{1}{a^{2}} & 0 \\
0 & \frac{1}{b^{2}}
\end{array}\right] .
$$

Thereby we have introduced a metric - the Zermelo metric - in each tangent plane $T_{(u, v)} \mathcal{U}$ in the parameter plane $\mathcal{U}$. Let $A=\left(\alpha_{1}, \alpha_{2}\right)$ and $B=\left(\beta_{1}, \beta_{2}\right)$ denote two vectors in the tangent plane with coordinates as stated with respect to the canonical basis. Then we apply $h_{(u, v)}$ as a metric in $T_{(u, v)} \mathcal{U}$ in the following way:

$$
h_{(u, v)}(A, B)=\left[\begin{array}{ll}
\alpha_{1} & \alpha_{2}
\end{array}\right] h_{(u, v)}\left[\begin{array}{l}
\beta_{1} \\
\beta_{2}
\end{array}\right] .
$$

The pair $\left(h_{(u, v)}, C(u, v)\right)$ is known as the Zermelo data for the corresponding ellipse field in the parameter domain. Specifically, with this data we can now express in a simple way the Hamilton orthogonality conditions for given vectors $V$ and $W$ in the tangent plane at the point $(u, v)$ :

1. $V$ is a position vector for a point on the ellipse $E_{(u, v)}$ if and only if $h_{(u, v)}(V-C, V-C)=1$ 
2. Suppose that $V$ is a position vector for a point $q$ on the ellipse $E_{(u, v)}$. Then $W$ is parallel to a tangent vector to the ellipse $E_{(u, v)}$ at the point $q$ if and only if $h_{(u, v)}(V-C, W)=0$. It follows from the strong convexity of the ellipse that for a given $W$ there are precisely two position vectors $V$ for points on the ellipse which both satisfy $h_{(u, v)}(V-C, W)=0$. Using the orientation of the parameter plane we get that there is one such $V$ to the right hand side of $W$ and the other lies to the left hand side of $W$.
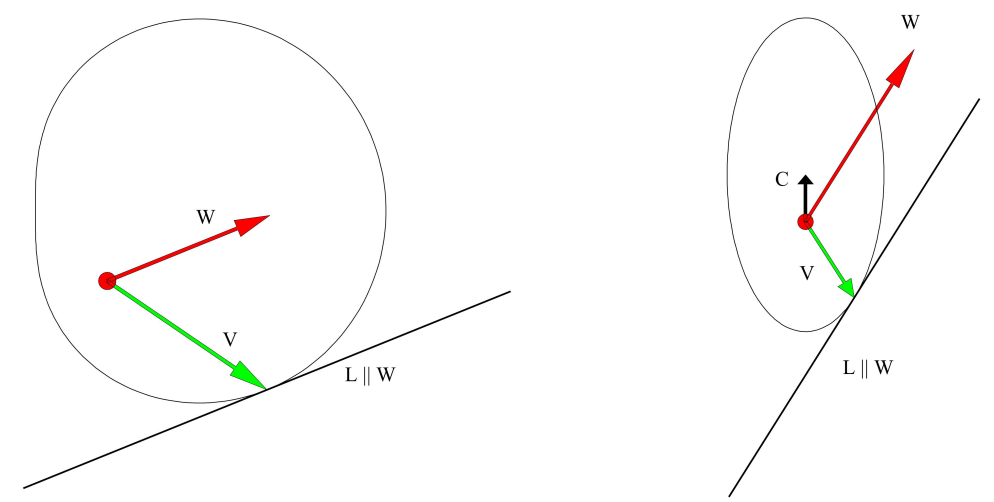

Figure 3: Given pointed indicatrices as shown, then in each case the $F$-unit vector to the right of the red fire line vector is constructed as the point on the indicatrix where the tangent is parallel to the fire line vector. The two vectors $V$ and $W$ are $F$-orthogonal. The vector $C$ is the wind shift contribution to the elliptic indicatrix.

We have therefore:

Corollary 8.1. For any wildfire $\gamma(s, t)$ in an elliptic indicatrix field $\mathcal{I}$, Hamilton orthogonality with respect to the corresponding Finsler metric $F$ can be stated in terms of Zermelo data $(h, C)$ as follows at every point $\gamma(s, t)=$ $(u, v)$.

$$
\begin{aligned}
h_{(u, v)}\left(\gamma_{t}^{\prime}(s, t)-C(u, v), \gamma_{t}^{\prime}(s, t)-C(u, v)\right) & =1 \\
h_{(u, v)}\left(\gamma_{t}^{\prime}(s, t)-C(u, v), \gamma_{s}^{\prime}(s, t)\right) & =0 .
\end{aligned}
$$

For elliptic template fields there is a direct way of getting the Finsler metric from the Zermelo data and vice versa: Suppose for example that we 
are given ellipse field data $a(u, v), b(u, v), C(u, v)=\left(c_{1}(u, v), c_{2}(u, v)\right)$, and $\theta(u, v)$, that is, we are given Zermelo data $(h, C)$. Then the corresponding Finsler metric $F$ is determined by the following expression, see e.g. [4, Section 1.1.2]:

$$
\begin{aligned}
& F(p, V)=F(u, v, x, y) \\
= & \left(\frac{\sqrt{\lambda(u, v) h_{(u, v)}(V, V)+h_{(u, v)}^{2}(V, C)}}{\lambda(u, v)}\right)-\left(\frac{h_{(u, v)}(V, C)}{\lambda(u, v)}\right),
\end{aligned}
$$

where

$$
\lambda(u, v)=1-h_{(u, v)}(C, C)>0
$$

The development of Randers spaces and their equivalent Zermelo data has an interesting history which is of particular relevance for the wildfire problems in elliptic template fields because - as we have seen - the so-called Zermelo (geodesic) navigation problem is essentially identical to the wildfire spread problem. We refer to $[48,49,50,47,51,4,52,53,54,8,55,56,57$, 5, 58, 59, 60] for details on Randers spaces and on the Zermelo navigation problem.

\section{Richards' equations}

In this section we observe how Richards' equations for the spread of elliptic wildfires fit naturally into the Finsler geodesic spray paradigm, and in particular that they are in fact equivalent to the Zermelo version of the Hamilton orthogonality conditions in Corollary 8.1.

Theorem 9.1 (Richards [20], Glasa and Halada [28]). A wildfire $\gamma(s, t)$ on a given ellipse template field with Zermelo equivalent data $a(u, v), b(u, v)$, $C(u, v)=\left(c_{1}(u, v), c_{2}(u, v)\right)$, and $\theta(u, v)$ is determined by the following equations for the partial derivatives $\gamma_{s}^{\prime}(s, t)=\left(u_{s}^{\prime}, v_{s}^{\prime}\right)$ and $\gamma_{t}^{\prime}(s, t)=\left(u_{t}^{\prime}, v_{t}^{\prime}\right)$ :

$$
\begin{aligned}
u_{t}^{\prime}= & \frac{a^{2} \cos (\theta)\left(u_{s}^{\prime} \sin (\theta)+v_{s}^{\prime} \cos (\theta)\right)-b^{2} \sin (\theta)\left(u_{s}^{\prime} \sin (\theta)+v_{s}^{\prime} \cos (\theta)\right)}{\sqrt{a^{2}\left(u_{s}^{\prime} \sin (\theta)+v_{s}^{\prime} \cos (\theta)\right)^{2}+b^{2}\left(u_{s}^{\prime} \cos (\theta)-v_{s}^{\prime} \sin (\theta)\right)^{2}}} \\
& +c_{1} \cos (\theta)+c_{2} \sin (\theta)
\end{aligned}
$$




$$
\begin{aligned}
v_{t}^{\prime}= & \frac{-a^{2} \sin (\theta)\left(u_{s}^{\prime} \sin (\theta)+v_{s}^{\prime} \cos (\theta)\right)-b^{2} \cos (\theta)\left(u_{s}^{\prime} \sin (\theta)+v_{s}^{\prime} \cos (\theta)\right)}{\sqrt{a^{2}\left(u_{s}^{\prime} \sin (\theta)+v_{s}^{\prime} \cos (\theta)\right)^{2}+b^{2}\left(u_{s}^{\prime} \cos (\theta)-v_{s}^{\prime} \sin (\theta)\right)^{2}}} \\
& -c_{1} \sin (\theta)+c_{2} \cos (\theta) .
\end{aligned}
$$

In particular, if the wind vector field $C(u, v)$ is directed in the positive direction of the $v$-axis we obtain by inserting $\theta=0$ :

$$
\begin{aligned}
& u_{t}^{\prime}=\frac{a^{2} v_{s}^{\prime}}{\sqrt{a^{2}\left(v_{s}^{\prime}\right)^{2}+b^{2}\left(u_{s}^{\prime}\right)^{2}}}+c_{1} \\
& v_{t}^{\prime}=\frac{-b^{2} u_{s}^{\prime}}{\sqrt{a^{2}\left(v_{s}^{\prime}\right)^{2}+b^{2}\left(u_{s}^{\prime}\right)^{2}}}+c_{2} .
\end{aligned}
$$

Remark 9.2. We note that this is a slight generalization of the original version of Richards' equations, since here we allow the (wind-) vector $C(u, v)$ to be not necessarily directed along one of the main axes of the ellipses in the field.

Proof. It is a straightforward calculation to see that with $\gamma(s, t)=(u(s, t), v(s, t))$, $\gamma_{s}^{\prime}(s, t)=\left(u_{s}^{\prime}, v_{s}^{\prime}\right)$ and $\gamma_{t}^{\prime}(s, t)=\left(u_{t}^{\prime}, v_{t}^{\prime}\right)$ as in (61) and (62) we get from (53) with $\theta=0$, that (55) and (56) are equivalent to (61) and (62). Since these relations are all tensorial, i.e. independent of the coordinate system, they also hold in the general setting of the theorem, i.e. for any value of $\theta$.

In short it therefore follows that:

Theorem 9.3. The wildfire solutions to Richards' equations are precisely the F-geodesic sprays obtained via the exponential map as in (42) - based on the Finsler metric obtained from the given elliptic template field as in (57).

\section{A simple example}

With the following simple choice of ellipse field, i.e. Zermelo data, we obtain the corresponding wildfire spread (from ignition at $(u, v)=(0,0)$ ) as indicated in figure 4:

$$
\begin{aligned}
a(u, v) & =1 \\
b(u, v) & =3 \\
C(u, v) & =(0,2) \\
\theta(u, v) & =u-(2 / 5) .
\end{aligned}
$$



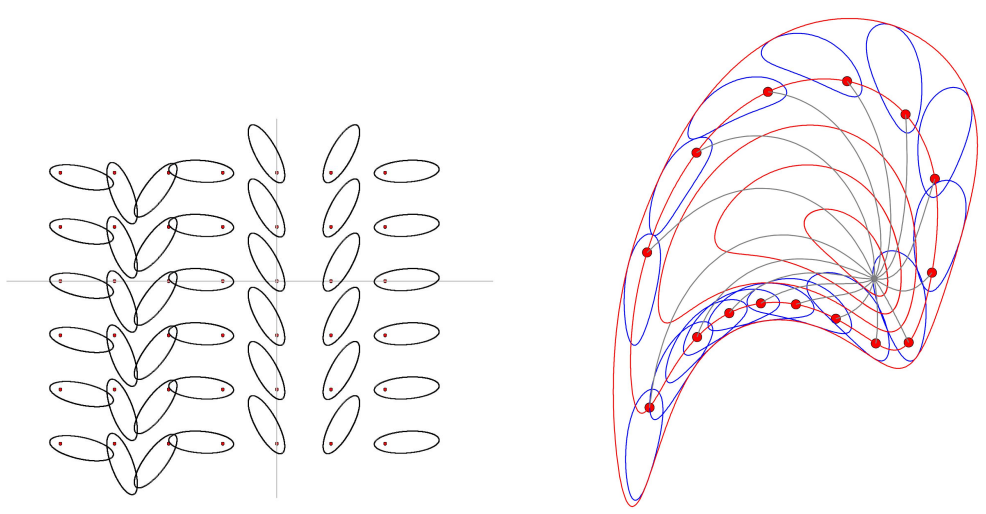

Figure 4: The elliptic fire templates at various points in the $(u, v)$-plane and the corresponding wildfire spread ignited at the center point $(u, v)=(0,0)$.

The figure has been constructed from a numerical solution of the geodesic spray equations for the $F$-exponential map in (42).

Remark 10.1. We observe in figure 5 (see also figure 8), that the F-geodesic fire discs centered at the points on the next-outermost fire line will envelope the outermost fire line, whereas the corresponding templates themselves clearly fail to solve this task. The reason for this discrepancy is the presence of curvature in the background Finsler metric, which - in figure 5 - is induced solely from the very simple rotation (by $\theta(u, v)$ ) of the rigid ellipse to obtain the non-constant template field. The fire templates are not objects in the curved parameter domain, they are linearized objects in the respective tangent planes. If the fire templates are applied directly in a stepwise approximate enveloping procedure, then they will tend to accumulate significant errors in comparison with the correct geodesic fronts of the geodesic spray as observed - but without the explanation and solution given here - in e.g. [15], [16], and [19].

Remark 10.2. Another important observation is, that the fire discs centered at the points on the next-outermost fire line clearly do not envelope the previous fire line just before (i.e. the third outermost fire line). This is but a clear 

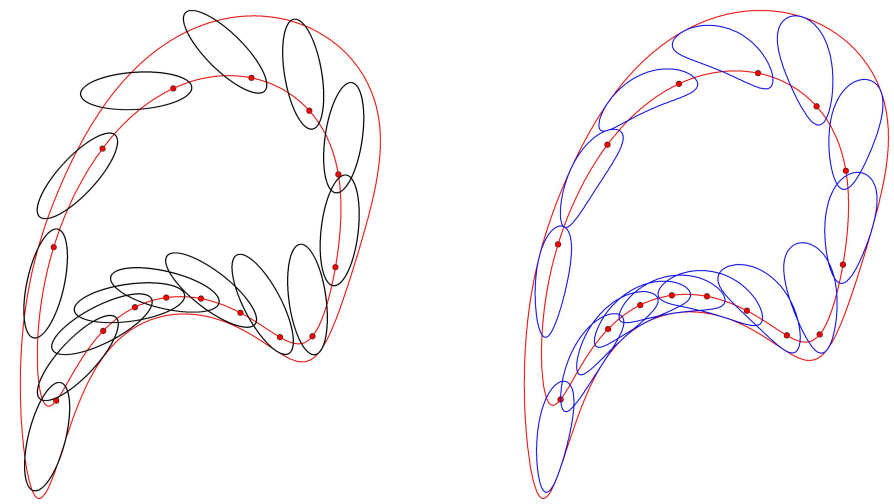

Figure 5: The exponential fire discs envelope the outer most fire front (from the given inner front). The elliptic template indicatrices do not envelope correctly.

token of the asymmetry and anisotropy of the typical Finsler metric background. Forwards moving geodesics do not necessarily have the same tracks or fronts as the corresponding backwards moving geodesics, see [11] and [10].

\section{A hemispherical elliptic wildfire}

The following example is based on a very recent work on concrete Randers spaces with constant curvature [61], where the corresponding wildfire problem has a simple analytic solution. It is of particular interest for us because the resulting wildfires in this metric are analytically solvable and thus they represent unique possibilities for comparing the analysis with the results of numerical methods and simulations that are applied to solve the wildfire spread problems. This example is thence one of the rare cases which offers a much needed benchmark situation for the previous numerical solutions to the wildfire problem, c.f. remark 10.1 above.

A hemisphere can be parametrized as follows

$$
S \quad: \quad r(u, v)=\left(u, v, \sqrt{1-u^{2}-v^{2}}\right) \quad, \quad(u, v) \in \mathcal{U},
$$

where $\mathcal{U}$ is the open unit disc in the $(u, v)$-plane. Openness is needed because this parametrization is obviously not regular at the equator of the sphere. 
We now construct explicit Zermelo data for a special elliptic template field on the hemisphere, stemming from a field of identical but shifted circles in the tangent planes to the hemisphere surface as indicated in figure 6 . All the circles have Euclidean radius $\alpha$. The only change from one point to another on the hemisphere is the shift of the circle in its respective tangent plane towards the east as the tangent plane is moved towards the equator. The field of shifted circles is rotationally symmetric with respect to rotation about the vertical axis through the North pole.
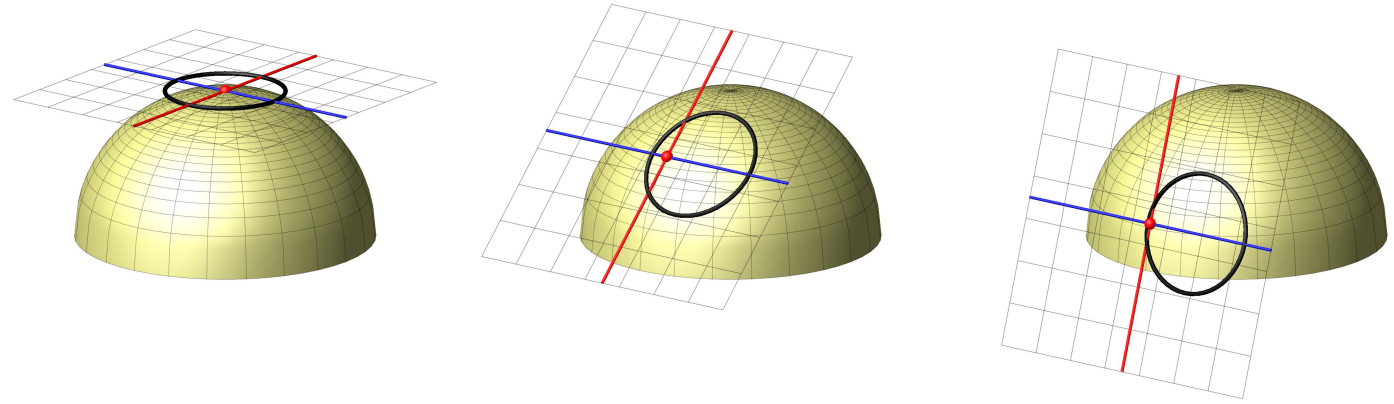

Figure 6: Tangent planes to the hemisphere surface with eastwards shifted circular fire templates.

In the parameter domain the circle field is then represented as a field of (projected) pointed ellipses with the following standard data, see figure 7:

$$
\begin{aligned}
a(u, v) & =\alpha \sqrt{1-u^{2}-v^{2}} \\
b(u, v) & =\alpha \\
C(u, v) & =\alpha \sqrt{u^{2}+v^{2}}(\sin (\theta(u, v)), \cos (\theta(u, v)))=\alpha(-v, u) \\
\theta(u, v) & =-\arg (u+i v) .
\end{aligned}
$$

The specific ellipse in the $\left\{\partial_{u}, \partial_{v}\right\}$ basis of $T_{(u, v)} \mathcal{U}$ at the point $(u, v)$ in the parameter disc domain $\mathcal{U}$ then has the following parametrization:

$$
\begin{aligned}
& E_{(u, v)}(\phi)= \\
& \frac{\alpha}{\sqrt{u^{2}+v^{2}}}\left[\begin{array}{l}
u \sqrt{1-u^{2}-v^{2}} \cos (\phi)-v \sin (\phi)-v \sqrt{u^{2}+v^{2}} \\
v \sqrt{1-u^{2}-v^{2}} \cos (\phi)+u \sin (\phi)+u \sqrt{u^{2}+v^{2}}
\end{array}\right] .
\end{aligned}
$$


Let $R(u, v)$ denote the rotation matrix:

$$
R(u, v)=\frac{1}{\sqrt{u^{2}+v^{2}}}\left[\begin{array}{cc}
u & v \\
-v & u
\end{array}\right]
$$

and let $(\xi, \eta)$ denote new coordinates as follows:

$$
\left[\begin{array}{l}
\xi \\
\eta
\end{array}\right]=R(u, v)\left[\begin{array}{l}
x \\
y
\end{array}\right]
$$

Then

$$
\left(\frac{\xi}{\alpha \sqrt{1-u^{2}-v^{2}}}\right)^{2}+\left(\frac{\eta-\alpha \sqrt{u^{2}+v^{2}}}{\alpha}\right)^{2}=1,
$$

and we get the following simple Zermelo data for the template field:

$$
h_{(u, v)}=\frac{1}{\alpha^{2}\left(1-u^{2}-v^{2}\right)}\left[\begin{array}{cc}
1-v^{2} & u v \\
u v & 1-u^{2}
\end{array}\right] \quad, \quad C(u, v)=\alpha(-v, u)
$$

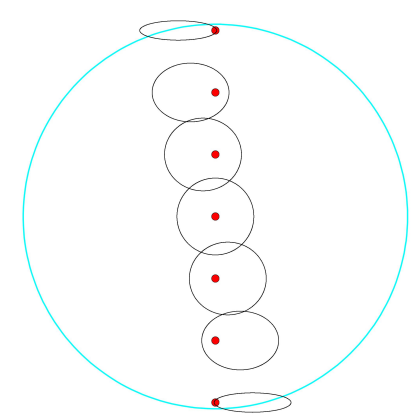

Figure 7: The hemisphere parameter plane with elliptic fire templates in the tangent planes at the indicated positions. All other templates are obtained by extension to the full line of the red points and rotation around the center of the shown disk.

The corresponding Finsler metric $F$ is also relatively simple. It can be obtained directly from (76) via (57):

$$
F(u, v, x, y)=\frac{\sqrt{x^{2}+y^{2}}-u y+v x}{\alpha\left(1-u^{2}-v^{2}\right)} .
$$



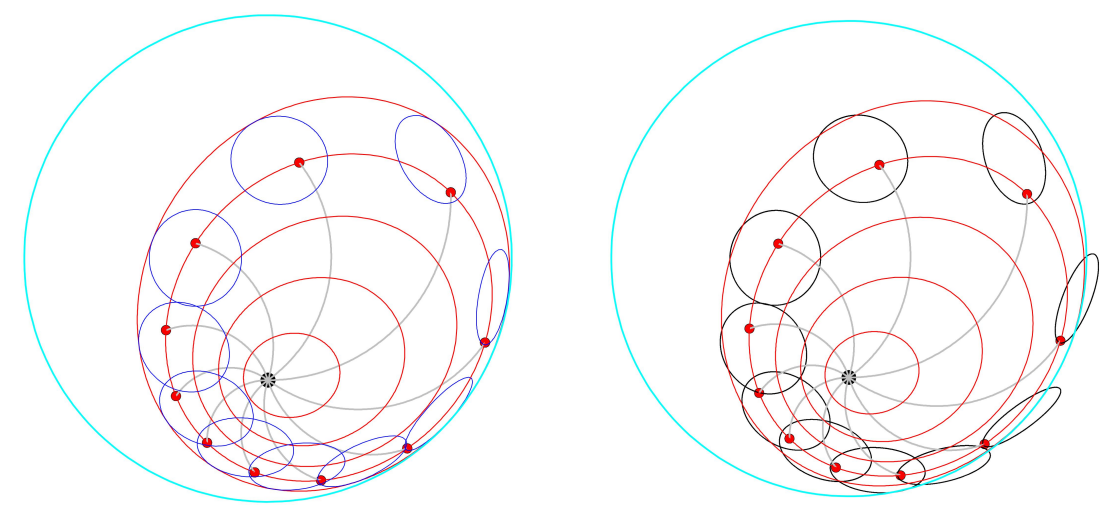

Figure 8: An $F$-geodesic spray for the hemisphere and a geodesic disc-enveloping of the outer front. The indicatrices do not themselves envelope correctly - in fact they even fall out of the unit circle domain.

This equation is much simpler than the corresponding quadratic equation. The advantages of using this format of the ellipse field will be discussed in the following sections. It corresponds precisely to the example discussed by Crampin and Mestdag in [61, Section 3]. They show that the F-geodesic equations in this case reduce to the extremely simple ones:

$$
\begin{aligned}
& \rho u^{\prime}(t)=-v(t)+\left(v_{0}+\rho x_{0}\right) \\
& \rho v^{\prime}(t)=u(t)-\left(u_{0}-\rho y_{0}\right),
\end{aligned}
$$

where $\rho=1 / 2 \alpha$ and the initial (ignition) data for the fire particle geodesics is $(u, v)=\left(u_{0}, v_{0}\right)$ and $\left(u^{\prime}(0), v^{\prime}(0)\right)=\left(x_{0}, y_{0}\right)$. Every $F$-geodesic solution is therefore a circle in the unit $(u, v)$-disk. They are all parametrized in the counterclockwise direction and they are tangent to the boundary of the unit parameter disc - at points from where they cannot be extended because the Finsler metric is clearly singular at the boundary of the disc.

When we assume ignition of the solution at the point $\left(0, v_{0}\right), v_{0}^{2}<1$, at time $t=0$ we get the following $F$-geodesic wildfire with that ignition point. (All other point-ignited solutions are obtained from such a solution by symmetry.) 


$$
\gamma(s, t)=(u(s, t), v(s, t))=\left(\frac{1-v_{0}^{2}}{2\left(1+v_{0} \cos (s)\right)}\right)(f(s, t), g(s, t))
$$

where

$$
\begin{aligned}
& f(s, t)=\cos (2 \alpha t) \sin (s)+\sin (2 \alpha t) \cos (s)-\sin (s), \\
& g(s, t)=\sin (2 \alpha t) \sin (s)+\cos (2 \alpha t) \cos (s)+\cos (s)+v_{0} .
\end{aligned}
$$

The solution tracks in the unit disc in the parameter plane is indicated by a few examples in figure 8 , and the corresponding hemispherical solutions (lifted via the parametrization $r$ ) are shown in figure 9.
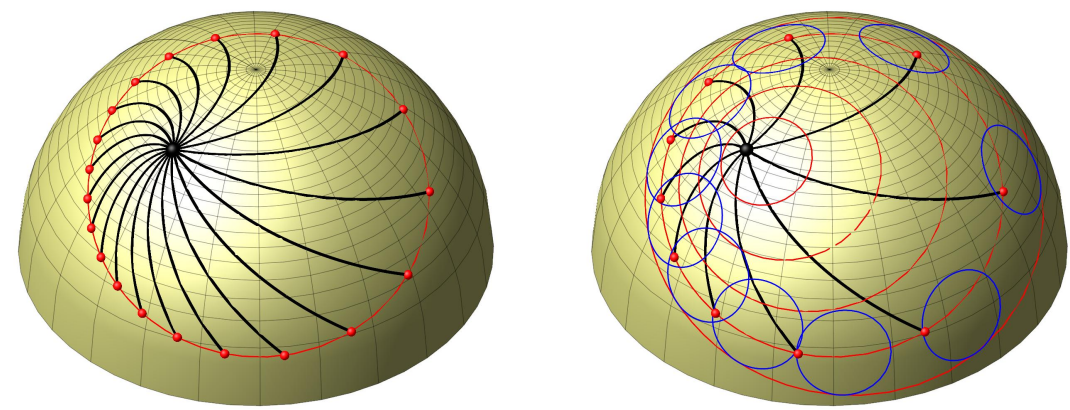

Figure 9: The lifted fire fronts to the hemisphere and an indication of Huyghens' envelope principle at work to mold the outer-most front line from the previous front line.

\section{A non-elliptic example}

There is, of course, an abundance of other strongly convex ovals in the plane, than just the ellipses, that can be used for setting up an indicatrix field - and thence a Finsler metric - in a given parameter domain; see e.g. the nice constructive approaches to the analysis of various relevant ovals in $[62,63,64]$ and the previous works towards the generalization of the elliptic fire-template fields in $[65,66,30]$. 


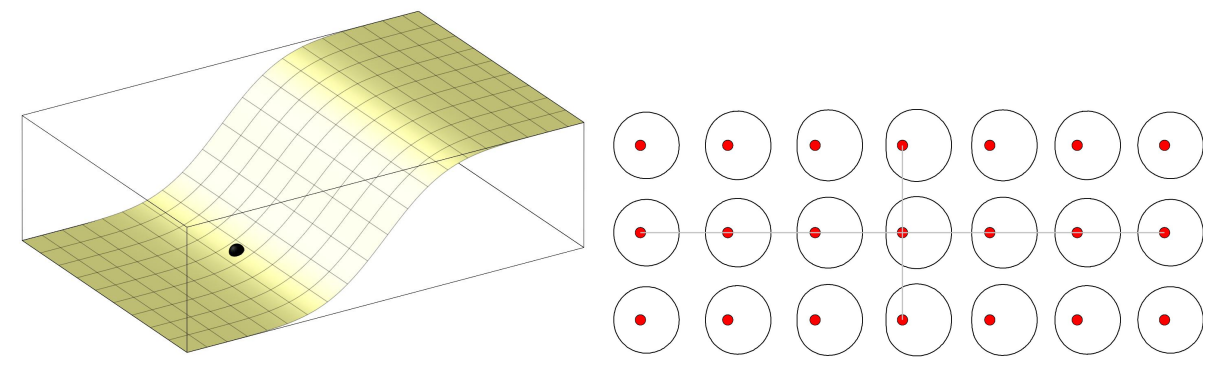

Figure 10: A simple sloped graph fuel surface (with a marked fire ignition point) over the $(u, v)$-plane and a corresponding Matsumoto type fire template field in the $(u, v)$-parameter domain.

The Matsumoto metric is a non-elliptic Finsler metric whose indicatrices are Pascal Limaçons with polar representation as follows

$$
\mathcal{M}: \rho(\phi)=1+d \cos (\phi) \quad, \quad d<1 / 2 .
$$

They can effectively be used for the construction of slope dependent Finsler metrics for fuel landscapes with topography. We may use $d$ as the slope dependent parameter. For example, if we consider the graph surface $r(u, v)=(u, v, \operatorname{erf}(u))$, as shown in figure 10, we get the slope function

$$
\sigma(u, v)=e^{-u^{2}}
$$

so we may apply the position dependent $d$-values $d(u)=(2 / 5) e^{-u^{2}}$ for the indicatrix template in the parameter tangent space at $(u, v)$ :

$$
\mathcal{M}_{(u, v)}: \rho(\phi)=1+d(u) \cos (\phi)
$$

An equation for this indicatrix is then:

$$
\mathcal{M}_{(u, v)}:\left(x^{2}+y^{2}-d(u) x\right)^{2}-x^{2}-y^{2}=0 \quad .
$$

Using the previously mentioned Okubo technique this equation can be recast into a 1-homogeneous version, which then gives the Finsler metric with $\mathcal{M}_{(u, v)}=F^{-1}(1)$, see e.g. [11, 63]:

$$
F(u, v, x, y)=\frac{\left(\sqrt{x^{2}+y^{2}}-d(u) x\right)\left(x^{2}+y^{2}\right)}{x^{2}+y^{2}-d^{2}(u) x^{2}}
$$


The template field is shown in figure 10. When we insert this Finsler metric $F$ into the geodesic equations for the $F$-geodesic exponential map in definition 7.1 , we obtain the wildfire solution ignited at $(u(0), v(0))=(-1,0)$ as indicated in figure 11. We observe that the uphill spread of the wildfire is much faster than the downhill and the horizontal spread - as it should be.
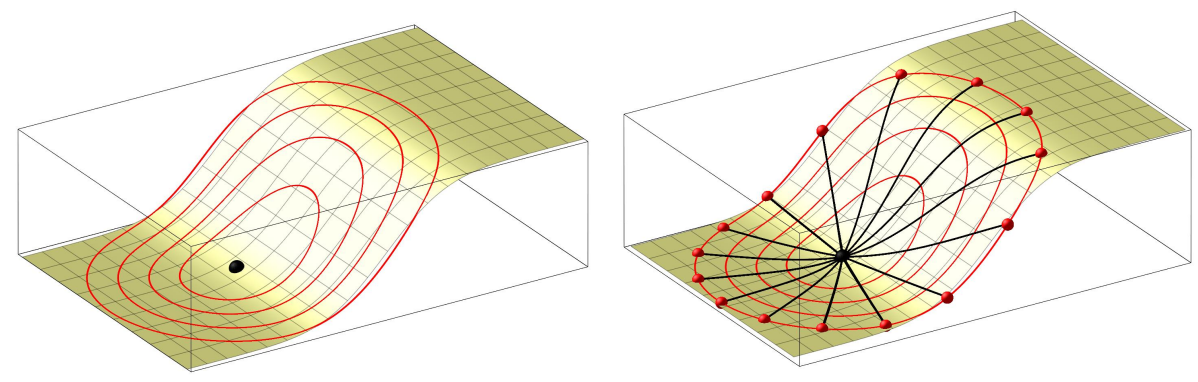

Figure 11: The spread of the Matsumoto type fire from the ignition point $(-1,0)$. To the right are shown a number of corresponding $F$-geodesic fire tracks for the resulting wildfire.

\section{Conclusion}

We have shown that each specific choice of a smoothly varying strongly convex pointed oval field (modelling small time linearized firelets) in the parameter fuel domain produces a Finsler metric $F$ with this given indicatrix field, and under the Huyghen's enveloping Ansatz the corresponding wildfires are governed by the $F$ geodesic spray equations.

Specifically, for the elliptic fire template fields we have embedded the well known Richards' equations into the Finsler geodesic spray paradigm:

Theorem 13.1. The wildfire solutions to Richards' equations are precisely the F-geodesic sprays obtained via the exponential map as in (42) - based on the Finsler metric obtained from the given elliptic template field as in (57).

Moreover, we have shown simple examples that illustrate the general theory and applicability of the $F$-geodesic spray paradigm - including one example with analytic wildfire solutions. 
In spite of their simplicity the chosen examples are presented in such a way that they also pinpoint the accumulation of errors that inevitably appears when the wildfire spread is directly constructed from the template field via a stepwise enveloping procedure without first using the needed F-geodesic exponential modification of the templates as per definitions 6.4 and 6.5, see remark 10.1.

\section{Discussion}

Our discussion is so far only semi-global in the sense that we do not in this paper consider the formation of cut loci, the so-called 'bear hugs', which typically appear during the long time spreading of wildfires in non-constant fuel domains. A beginning discussion of these aspects of global Finsler geometry and their possible applications for the wildfire spread modelling can be found in $[67,68]$.

Even in the semi-global regular setting the accumulation of errors, which is induced by using the template field more or less directly to envelope the next time step front line, needs further analysis and comparison with the geodesic wildfire spread mechanism that we have applied in this paper, see $[15,16,19]$. This issue has much to do with the curvature sensitivity of the geodesic spray, i.e. the study of Jacobi fields and the flag curvatures along the geodesic fire tracks.

It is fairly straightforward to generalize the Finsler geodesic spray paradigm to include time varying fuel and meteorological data as well as higher dimensions into our study of wildfire spreads. Dimension 3, which includes the height parameter from ground to canopy, is, of course, the most interesting and most relevant for this particular real world phenomenon.

These aspects will be taken up in future work by the present author and/or by other authors.

\subsection{Acknowledgement}

The author would like to thank the referees for encouraging comments and for suggestions which have improved the presentation of this work. 


\section{Bibliography}

[1] P. L. Antonelli, R. S. Ingarden, M. Matsumoto, The theory of sprays and Finsler spaces with applications in physics and biology, Vol. 58 of Fundamental Theories of Physics, Kluwer Academic Publishers Group, Dordrecht, 1993.

[2] P. L. Antonelli, A. Bóna, M. A. Slawiński, Seismic rays as Finsler geodesics, Nonlinear Anal. Real World Appl. 4 (5) (2003) 711-722. doi : 10.1016/S1468-1218(02)00073-1.

[3] T. Yajima, H. Nagahama, Finsler geometry of seismic ray path in anisotropic media, Proceedings of the Royal Society A - Mathematical Physical and Engineering Sciences 465 (2106) (2009) 1763-1777. doi:10.1098/rspa.2008.0453.

[4] D. Bao, C. Robles, Z. Shen, Zermelo navigation on Riemannian manifolds, J. Differential Geom. 66 (3) (2004) 377-435.

[5] M. Cvetič, G. W. Gibbons, Graphene and the Zermelo optical metric of the BTZ black hole, Ann. Physics 327 (11) (2012) 2617-2626. doi: 10.1016/j.aop. 2012.05.013.

[6] G. W. Gibbons, J. Gomis, C. N. Pope, General very special relativity is Finsler geometry, Phys. Rev. D 76 (8) (2007) 081701, 5. doi:10.1103/ PhysRevD.76.081701.

[7] L. Astola, L. Florack, Finsler geometry on higher order tensor fields and applications to high angular resolution diffusion imaging, International Journal of Computer Vision 92 (3) (2011) 325-336. doi: 10.1007/s11263-010-0377-z.

[8] E. Caponio, M. Angel Javaloyes, A. Masiello, On the energy functional on Finsler manifolds and applications to stationary spacetimes, Mathematische Annalen 351 (2) (2011) 365-392. doi:10.1007/ s00208-010-0602-7.

[9] T. Yajima, H. Nagahama, Finsler geometry for nonlinear path of fluids flow through inhomogeneous media, Nonlinear Analysis: Real World Applications 25 (2015) 1-8. doi:10.1016/j.nonrwa.2015.02.009. 
[10] Z. Shen, Lectures on Finsler geometry, World Scientific Publishing Co., Singapore, 2001. doi:10.1142/9789812811622.

[11] D. Bao, S.-S. Chern, Z. Shen, An introduction to Riemann-Finsler geometry, Vol. 200 of Graduate Texts in Mathematics, Springer-Verlag, New York, 2000.

[12] C. Wiedinmyer, J. Neff, Estimates of $\mathrm{CO}_{2}$ from fires in the United States: Implications for carbon management, Carbon Balance and Management 2 (1) (2007) 10.

[13] J. G. Goldammer, C. de Ronde (Eds.), Wildland Fire Management Handbook for Sub-Sahara Africa, A publication of the Global Fire Monitoring Center (GFMC), 2004.

[14] C. Tymstra, R. W. Bryce, B. M. Wotton, S. W. Taylor, O. B. Armitage, Development and Structure of Prometheus: the Canadian Wildland Fire Growth Simulation Model, Information Report NOR-X-417, Natural Resources Canada, Canadian Forest Service, Northern Forestry Centre, Edmonton, Alberta, Canada (2010).

[15] W. Cui, A. H. Perera, A study of simulation errors caused by algorithms of forest fire growth models, Forest Research Report 167, Ontario Forest Research Institute, Ontario Ministry of Natural Resources, Sault Ste. Marie, ON, Canada (2008).

[16] G. D. Papadopoulos, F.-N. Pavlidou, A comparative review on wildfire simulators, IEEE Systems Journal 5 (2) (2011) 233-243. doi:10.1109/ JSYST . 2011.2125230.

[17] A. L. Sullivan, Wildland surface fire spread modelling, 1990-2007. 1: Physical and quasi-physical models, International Journal of Wildland Fire 18 (4) (2009) 349-368. doi:10.1071/WF06143.

[18] A. L. Sullivan, Wildland surface fire spread modelling, 1990-2007. 2: Empirical and quasi-empirical models, International Journal of Wildland Fire 18 (4) (2009) 369-386. doi:10.1071/WF06142.

[19] A. L. Sullivan, Wildland surface fire spread modelling, 1990-2007. 3: Simulation and mathematical analogue models, International Journal of Wildland Fire 18 (4) (2009) 387-403. doi:10.1071/WF06144. 
[20] G. D. Richards, Elliptical growth model of forest fire fronts and its numerical solution, International Journal for Numerical Methods in Engineering 30 (6) (1990) 1163-1179.

[21] G. D. Richards, Properties of elliptical wildfire growth for time dependent fuel and meteorological conditions, Combustion Science and Technology 92 (1-3) (1993) 145-171.

[22] G. D. Richards, A general mathematical framework for modeling 2dimensional wildland fire spread, International Journal of Wildland Fire 5 (2) (1995) 63-72.

[23] G. D. Richards, R. W. Bryce, A computer algorithm for simulating the spread of wildland fire perimeters for heterogeneous fuel and meteorological conditions, International Journal of Wildland Fire 5 (2) (1995) $73-79$.

[24] G. D. Richards, The mathematical modelling and computer simulation of wildland fire perimeter growth over a 3-dimensional surface, International Journal of Wildland Fire 9 (3) (1999) 213-221.

[25] C. E. Van Wagner, A simple fire-growth model, For. Chron. 45 (1969) 103-104.

[26] D. L. Anderson, E. A. Catchpole, N. J. De Mestre, T. Parkes, Modelling the spread of grass fires, J. Austral. Math. Soc. (Series B) 23 (1982) 451466 .

[27] J. Glasa, L. Halada, Application of envelope theory for 2D fire front evolution, Forest Ecology and Management 234 (2006) S129. doi:10. 1016/j.foreco.2006.08.170.

[28] J. Glasa, L. Halada, On elliptical model for forest fire spread modeling and simulation, Mathematics and Computers in Simulation 78 (1) (2008) 76-88. doi:10.1016/j.matcom.2007.06.001.

[29] J. Glasa, L. Halada, On mathematical foundations of elliptical forest fire spread model, Forest Fires: Detection, Suppression and Prevention, For. Fires: Detect., Suppr. and Prev (2009) 315-334. 
[30] J. Glasa, L. Halada, A note on mathematical modelling of elliptical fire propagation, Computing and Informatics, Comput. Inf 30 (6) (2011) $1303-1319$.

[31] J. Glasa, P. Weisenpacher, L. Halada, Analysis of forest fire behaviour by advanced computer fire simulators, Komunikacie 13 (2) (2011) 26-31.

[32] O. Rios, W. Jahn, G. Rein, Forecasting wind-driven wildfires using an inverse modelling approach, Natural Hazards and Earth System Sciences, Vol 14, Iss 6, Pp 1491-1503 (2014).

[33] V. I. Arnol'd, Mathematical methods of classical mechanics, 2nd Edition, Vol. 60 of Graduate Texts in Mathematics, Springer-Verlag, New York, 1989, translated from the Russian by K. Vogtmann and A. Weinstein. doi:10.1007/978-1-4757-2063-1.

[34] N. Innami, Generalized metrics for second order equations satisfying Huygens' principle, Nihonkai Math. J. 6 (1) (1995) 5-23.

[35] N. Innami, The class of second order equations which Riemannian geometry can be applied to, J. Math. Soc. Japan 45 (1) (1993) 89-103. doi:10.2969/jmsj/04510089.

[36] R. C. Rothermel, A mathematical model for predicting fire spread in wildland fuels, USDA Forest Service, 1972, Research Paper INT-115, Ogden, Utah, USA.

[37] R. C. Rothermel, How to predict the spread and intensity of forest and range fires, General Technical Report INT-143, U.S. Department of Agriculture, Forest Service, Intermountain Forest and Range Experiment Station, Ogden, UT (1983).

[38] D. X. Viegas, Forest fire propagation, Philosophical Transactions Mathematical Physical and Engineering Sciences 356 (1748) (1998) 2907.

[39] J. André, D. Viegas, J. Urbano, Forest fire spread models: The local quasi-equilibrium approach, Combustion Science and Technology 178 (12) (2006) 2115-2143. doi :10.1080/00102200600910841.

[40] J. C. S. André, J. C. Gonçalves, G. C. Vaz, D. X. Viegas, Angular variation of fire rate of spread, International Journal of Wildland Fire 22 (7) (2013) 970-979. doi:10.1071/WF12028. 
[41] J. Margerit, O. Séro-Guillaume, Modelling forest fires. Part ii: reduction to two-dimensional models and simulation of propagation, International Journal of Heat and Mass Transfer 45 (8) (2002) 1723-1737. doi:10. 1016/s0017-9310(01)00249-6.

[42] O. Séro-Guillaume, S. Ramezani, J. Margerit, D. Calogine, On large scale forest fires propagation models, International Journal of Thermal Sciences 47 (6) (2008) 680-694. doi:10.1016/j.ijthermalsci.2007. 06.016.

[43] B. Riemann, H. Weyl, Über die Hypothesen welche der Geometrie zu Grunde liegen, 2. Aufl, Springer, 1921.

[44] D. Struik, Lectures on Classical Differential Geometry, Dover Publications, 1988.

[45] W. Klingenberg, A Course in Differential Geometry, Springer, 1978.

[46] I. Bucataru, M. Slawinski, Generalized orthogonality between rays and wavefronts in anisotropic inhomogeneous media, Nonlinear Analysis Real World Applications 6 (1) (2005) 111-121. doi : 10.1016/j.nonrwa. 2004.03 .004$.

[47] G. Randers, On an asymmetrical metric in the fourspace of general relativity, Phys. Rev. (2) 59 (1941) 195-199.

[48] E. Zermelo, Über das Navigationsproblem bei ruhender oder veränderlicher Windverteilung, ZAMM - Zeitschrift Für Angewandte Mathematik und Mechanik 11 (2) (1931) 114-124. doi:10.1002/zamm. 19310110205.

[49] T. Levi-Civita, Über Zermelo's Luftfahrtproblem, ZAMM - Zeitschrift Für Angewandte Mathematik und Mechanik 11 (4) (1931) 314-322. doi : 10.1002/zamm. 19310110404.

[50] R. v. Mises, Zum Navigationsproblem der Luftfahrt, ZAMM - Zeitschrift Für Angewandte Mathematik und Mechanik 11 (5) (1931) 373-381. doi:10.1002/zamm.19310110505.

[51] C. Robles, Geodesics in Randers spaces of constant curvature, Trans. Amer. Math. Soc. 359 (4) (2007) 1633-1651 (electronic). doi:10.1090/ S0002-9947-06-04051-7. 
[52] Q. Xia, On the flag curvature of a class of Randers metric generated from the navigation problem, Journal of Mathematical Analysis and Applications 397 (1) (2013) 415-427. doi:10.1016/j.jmaa.2012.07. 035 .

[53] L. Huang, X. Mo, On geodesics of Finsler metrics via navigation problem, Proceedings of the American Mathematical Society 139 (8) (2011) 3015 .

[54] E. Caponio, M. A. Javaloyes, M. Sánchez, On the interplay between Lorentzian causality and Finsler metrics of Randers type, Rev. Mat. Iberoam. 27 (3) (2011) 919-952. doi:10.4171/RMI/658.

[55] M. Crampin, Randers spaces with reversible geodesics, Publicationes Mathematicae-Debrecen 67 (3-4) (2005) 401-409.

[56] T. Mestdag, On the geometry of Randers manifolds, Reports on Mathematical Physics 50 (2) (2002) 167-193. doi:10.1016/s0034-4877(02) 80053-2.

[57] X. Cheng, Z. Shen, Finsler geometry : An approach via Randers spaces, Springer, Science Press, 2012.

[58] G. W. Gibbons, C. A. R. Herdeiro, C. M. Warnick, M. C. Werner, Stationary metrics and optical Zermelo-Randers-Finsler geometry, Phys. Rev. D 79 (4) (2009) 044022, 21. doi:10.1103/PhysRevD.79.044022.

[59] G. W. Gibbons, C. M. Warnick, Traffic noise and the hyperbolic plane, Ann. Physics 325 (4) (2010) 909-923. doi:10.1016/j.aop.2009.12. 007.

[60] E. Caponio, M. A. Javaloyes, M. Sánchez, Wind Finslerian structures: From Zermelo's navigation to the causality of spacetimes, arXiv:1407.5494 [math.DG] (2014) 75.

[61] M. Crampin, T. Mestdag, A class of Finsler surfaces whose geodesics are circles, Publicationes Mathematicae-Debrecen 84 (1-2) (2014) 3-16. doi:10.5486/PMD . 2014.5845.

[62] M. Matsumoto, A slope of a mountain is a Finsler surface with respect to a time measure, J. Math. Kyoto Univ. 29 (1) (1989) 17-25. 
[63] H. Shimada, S. V. Sabau, Introduction to Matsumoto metric, Nonlinear Analysis - Theory Methods and Applications 63 (5-7) (2005) E165-E168. doi:10.1016/j.na.2005.02.062.

[64] J. C. Fisher, Curves of constant width from a linear viewpoint, Mathematics Magazine 60 (3) (1987) 131-140. doi:10.2307/2689558.

[65] D. Green, A. Gill, I. Noble, Fire shapes and the adequacy of fire-spread models, Ecological Modelling 20 (1) (1983) 33-45.

[66] D. G. Green, Shapes of simulated fires in discrete fuels, Ecological Modelling 20 (1) (1983) 21-32.

[67] S. Markvorsen, From PA(X) to RPAM(X), in: E. Behrends, N. Crato, J. F. Rodrigues (Eds.), Raising Public Awareness of Mathematics, Springer, 2012.

[68] M. Tanaka, S. V. Sabau, The cut locus and distance function from a closed subset of a Finsler manifold, arXiv:1207.0918v3 [MathDG]. 